\title{
Choosing to Stop: Responses Evoked by Externally Triggered and Internally Generated Inhibition Identify a Neural Mechanism of Will
}

\author{
Jim Parkinson ${ }^{1,2,3}$ and Patrick Haggard ${ }^{1}$
}

\begin{abstract}
Inhibiting inappropriate action is key to human behavioral control. Studies of action inhibition largely investigated external stop signals, yet these are rare in everyday life. Instead healthy adults exert "self-control," implying an ability to decide internally to stop actions. We added "choose for yourself" stimuli to a conventional go/no-go task to compare reactive versus intentional action and inhibition. No-go reactions showed the N2 EEG potential characteristic of inhibiting prepotent motor responses, whereas go reactions did not. Interestingly, the N2
\end{abstract}

\section{INTRODUCTION}

Healthy human adults are thought to exert volitional control over their own actions. The flexibility and reasonsresponsiveness of voluntary action is a remarkable product of brain evolution and development. It underlies the notion of responsibility and is thus a necessary precursor of social living. Earlier neuroscientific work on volition focused on the conscious experience of initiating action (Libet, Gleason, Wright, \& Pearl, 1983), but recent studies emphasize instead the inhibitory elements of volition, linking it to self-control. Self-control involves the capacity to inhibit an action despite strong internal urges or external cues to act (Filevich, Kühn, \& Haggard, 2012). Animals that lack this capacity are effectively prisoners of their immediate environment (Shadlen \& Gold, 2004). Prepotent responses to immediate stimuli may need to be suppressed in order to achieve other goals, to conserve energy, to prevent harmful side effects of action, or to achieve greater benefits at a later date.

Self-control, therefore, implies the capacity for voluntarily inhibiting motor output. An important neurological model of self-control comes from utilization behaviors in patients with frontal damage (Boccardi, Della Salla, Motto, \& Spinnler, 2002; Lhermitte, 1983). These patients show an inability to suppress stimulus-driven actions afforded by their immediate environment. The presence of a glass on the table compels them to drink, even when

\footnotetext{
${ }^{1}$ University College London, ${ }^{2}$ Sackler Centre for Consciousness Science, ${ }^{3}$ University of Sussex
}

component was present for intentional choices both to act and also to inhibit. Thus, free choices involved a first step of intentionally inhibiting prepotent responses before generating or withholding an action. Intentional inhibition has a crucial role breaking the flow of stimulus-driven responding, allowing expression of volitional decisions. Even decisions to initiate selfgenerated actions require this prior negative form of volition, ensuring the "freedom from immediacy" characteristic of human behavior. they are not thirsty. In the dramatic example of unilateral "anarchic hand" (Kritikos, Breen, \& Mattingley, 2005; Della Sala, Marchetti, \& Spinnler, 1991), the unsuppressed stimulus-driven actions of the affected hand interfere with their ability to achieve voluntary action goals with the unaffected hand (McBride, Sumner, Jackson, Bajaj, \& Husain, 2013).

Some forms of self-control are tonic. For example, the decision to refrain from using an addictive drug must be maintained over a long period (Bjork \& Gilman, 2013). However, even tonic self-control ultimately involves the ability to inhibit urges "in the moment," implying a transient suppression or braking of prepotent action plans. Developmental studies of self-control in healthy children (Mischel, Ebbesen, \& Zeiss, 1972) show that inhibiting a rewarding stimulus-driven action, such as eating marshmallows, ultimately reduces to stopping the movement of the hand toward the plate. Many people recognize the feeling of "just stopping oneself at the last moment," for example, from saying an unkind word to a colleague. We use the term intentional inbibition to refer to such volitional braking of a prepotent action on the brink of execution (Filevich et al., 2012; Kühn, Haggard, \& Brass, 2009; Brass \& Haggard, 2007).

Go/no-go tasks offer a standard experimental paradigm for studying inhibition, although they may not capture the full capacity for self-control. When a go stimulus is sufficiently frequent, the action is said to be prepotent, and occasional no-go stimuli then require effortful inhibition of the motor response. ERP studies identified an N2 
component, occurring approximately 200 msec after no-go stimuli, that was stronger than the corresponding component following go stimuli (Pfefferbaum, Ford, Weller, \& Kopell, 1985). The N2 has been proposed as a neural marker of motor inhibition (Bokura, Yamaguchi, \& Kobayashi, 2001; Falkenstein, Hoormann, \& Hohnsbein, 1999; Eimer, 1993; Pfefferbaum et al., 1985), but others have suggested that it could reflect conflict between more and less frequent responses or effortful switching to a less common response (Randall \& Smith, 2011; Enriquez-Geppert, Konrad, Pantev, \& Huster, 2010; Smith, Smith, Provost, \& Heathcote, 2010; Donkers \& van Boxtel, 2004; Nieuwenhuis, Yeung, van den Wildenberg, \& Ridderinkhof, 2003).

We hypothesized that intentional inhibition would also involve inhibition of a prepotent response and would therefore evoke a strong no-go N2. We created a novel task for studying intentional inhibition (see also Parkinson \& Haggard, 2014) by randomly intermixing conventional go and no-go trials with free choice trials on which participants should freely select for themselves whether to act or inhibit (see Figure 1 for design details). This design allowed us to factorially compare action and inhibition for both reactive and intentional responses. To ensure a prepotent drive to act, reactive go trials were more frequent than other types.

\section{EXPERIMENTAL PROCEDURES}

\section{Participants}

Participants were recruited from an institutional participant database, were paid $£ 15$ for a maximum of $2.5 \mathrm{hr}$ experimental time, and were tested with local ethical committee approval. Twenty-one participants were tested in Experiment 1, and five were rejected on exclusion criteria detailed in the Procedure section below. For the 16 remaining (nine women), analyzed participants, mean age was 24.6 years $(S D=4.0)$. Twenty-two participants were tested in Experiment 2, and six were subsequently rejected. For the 16 remaining (10 women), analyzed participants, mean age was 22.7 years $(S D=3.3)$. All participants who tested were right-handed by self-report, had no neurological disorders, and had normal or corrected-to-normal vision.

\section{Procedure}

\section{Experimental Design}

The main experiment consisted of 720 trials in total. Participants responded to visual target arrows that followed a fixation cross (see Figure 1 for stimulus representation and timings). Stimuli were presented using the Psychophysics Toolbox 3 (Kleiner, Brainard, \& Pelli, 2007; Brainard, 1997; Pelli, 1997) in Matlab on a CRT monitor. Participants were given written instructions, later reiterated verbally by experimenter JP. They were instructed to prepare a righthand index finger button press, beginning preparation from the onset of each fixation cross, and to press the button as quickly as possible when they saw the reactive go target (two rightward pointing arrows), within the 706-msec response window. A small gray box appeared to confirm a successful response, which remained onscreen for the duration of the response window. If the response was too slow, the screen flashed red for $100 \mathrm{msec}$ after the response window to indicate an error. If they saw the reactive no-go target (two leftward pointing arrows), they were to withhold the prepared response and wait until the response window ended. The screen flashed red if they failed to inhibit.
Figure 1. Stimulus sequences used in Experiments 1 and 2. Notice the different percentages of each trial type in Experiments 1 and 2. Participants used their dominant (right) hand throughout, responding using a single button. Rightward arrows instructed a go response (reactive action). Leftward arrows instructed a no-go (withhold response; reactive inhibition). Diamond shapes indicated free choice trials (participants freely chose choice to act or withhold, corresponding to intentional action or intentional inhibition)

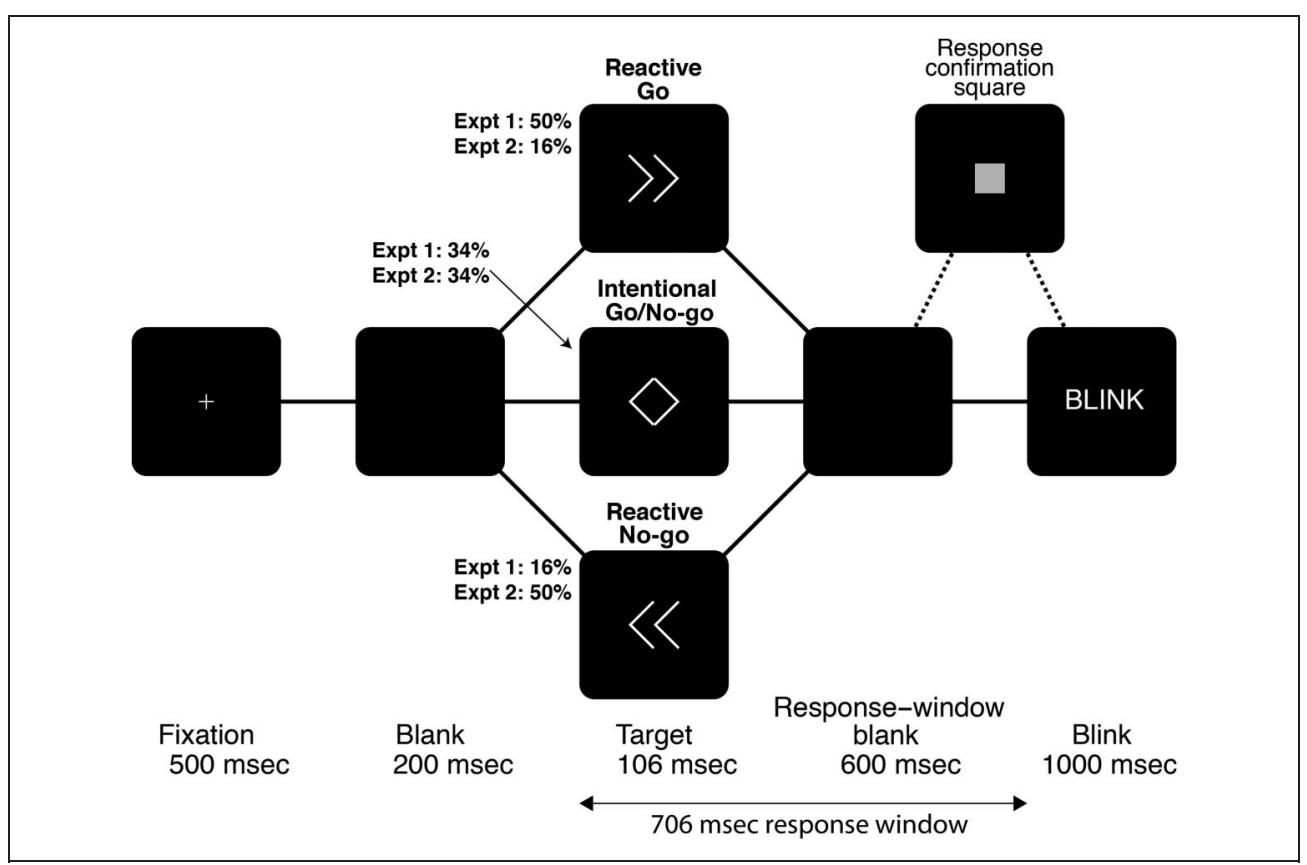


Participants were instructed that, if they saw the diamond shape stimulus, they should make an intentional choice to act or inhibit. Instructions for these trials were very specific and detailed: Participants were told that there was no right or wrong answer for these trials and there was no reward or punishment, nor did the experiment schedule change in any way, as a result of their choices. They were encouraged to make a quick spontaneous choice every time they saw the intentional target, without deciding in advance what that choice would be. The speed of the choice was emphasized, as the response window was just as fast as in reactive go trials, and so they were encouraged to not deliberate excessively on the choice. They were encouraged to choose actions and inhibitions "roughly equally" over the experiment, but without using strategies such as alternation or counting response numbers. These instructions aimed to mitigate some of the difficulties previously noted when studying voluntary choices in a constrained laboratory setting. Specifically, we wished to allow participants a free choice in their responses on one hand while avoiding automatized patterns of responding or predecision on what free choice to make before the imperative stimulus. We also needed to constrain "free" choice by the necessity of having enough trials to analyze in each condition. The instructions presented here represented a compromise between these different demands. The instructions were repeated verbally by the experimenter JP, who then confirmed the participant understood.

To minimize muscle-related artifacts in the EEG, participants were instructed to refrain from blinking, eye movements, and any other facial movement from the moment a fixation appeared until the word "BLINK" appeared onscreen to mark the beginning of a 1 -sec blink period between trials.

In Experiment 1, 360 trials (50\%) were reactive go (rightward arrows), 120 (16\%) were reactive no-go (leftward arrows), and 240 (34\%) were intentional choice trials (diamond shapes). The proportion of reactive go to no-go trials was reversed in Experiment 2 (16\%/50\%), whereas the proportion of intentional choice trials remained the same (34\%). Both experiments were split into 12 blocks of 60 trials each with self-timed breaks in between. Participants performed one extra block of practice trials before starting, which were not analyzed. Data acquisition lasted around $50 \mathrm{~min}$ in total.

\section{EEG Measurement}

EEG was measured using two 16-channel g.USBamp amplifiers (g.tec Medical Engineering GmbH, Schiedlberg, Austria), with active $\mathrm{Ag} / \mathrm{AgCl}$ electrodes. Twenty-six scalp electrodes were used corresponding to a sparse 1020 map, with the reference taken from the right earlobe. There were two additional electrodes on the left and right mastoids for subsequent re-referencing and two placed above and below the left eye for EOG mea- surements. Data were recorded at $512 \mathrm{~Hz}$ and filtered online with $0.1 \mathrm{~Hz}$ high-pass and $100 \mathrm{~Hz}$ low-pass hardware filters, with a notch filter around $50 \mathrm{~Hz}$ to remove electrical noise. The EEG recording also incorporated stimulus trigger markers for fixation, target and blink period onsets, and button responses.

\section{EEG Preprocessing}

All EEG processing was performed in Matlab utilizing the FieldTrip toolbox (Oostenveld, Fries, Maris, \& Schoffelen, 2011) and the author JP's custom scripts. Scalp EEG was re-referenced to linked mastoids, filtered using $0.3 \mathrm{~Hz}$ high-pass and $30 \mathrm{~Hz}$ low-pass filters, epoched from $200 \mathrm{msec}$ before target onset (baseline period) to $700 \mathrm{msec}$ afterwards, and demeaned over the baseline period to target onset. Upper and lower EOG channels were extracted from the data set, filtered using $0.5 \mathrm{~Hz}$ high-pass and $15 \mathrm{~Hz}$ lowpass two-pass Butterworth filters, and subtracted to create a single bipolar signal. This was analyzed for eye blink and movement artifacts exceeding $\pm 70 \mu \mathrm{V}$ in magnitude. Trials with artifacts from -100 to +700 msec relative to target onset were rejected.

Behavioral data were analyzed to detect errors in reactive trials, that is, reactive go trials in which no response was made within the response window (misses) and reactive no-go trials in which a response was made (false alarms). These trials were then removed from analysis. The remaining EEG trials were classified into four conditions: reactive go, reactive no-go, intentional go, and intentional no-go.

\section{Participant Exclusion Criteria}

Participants were excluded for two reasons. First, we computed the proportion of intentional go trials participants made within all intentional trials or the intentional action rate. Participants with intentional action rates less than $25 \%$ or greater than $75 \%$ were excluded to try to insure that no participants had a large bias toward intentional action or inhibition. Second, if a participant produced fewer than 50 nonerror trials in any condition (i.e., trials without artifacts or behavioral errors), they were also rejected. In fact, these two exclusion criteria tended to be mutually supportive. Five participants in Experiment 1 and six in Experiment 2 were rejected, leaving 16 participants in each experiment. Median RTs for reactive and intentional go trials were computed for each participant in each condition in each experiment.

\section{N2 Peak Amplitude Measurement}

To identify and measure the N2 components for each condition, the following procedure was performed in each participant: For each participant, channel-condition average EEG waveforms were computed for each channel (Fz, FCz, Cz; see Experiment 1 Results for justification) 
and condition combination. The N2 component was identified as the negative peak in each channel-condition average in the time range 150-300 msec after target onset. Mean N2 amplitude was calculated for each channelcondition combination by averaging EEG amplitude in a window 30 msec either side of each channel-condition peak. This procedure meant that mean N2 amplitude measurements were independent of latency differences between participants, conditions, or channels. The channelcondition N2 mean amplitudes were then averaged over the three channels to produce a condition N2 mean amplitude for each condition. These measures were used in the analyses.

\section{RESULTS}

In intentional trials, the mean rate at which participants freely chose to act was $55.1 \%(S D=12.3)$. Participants responded more rapidly on reactive go trials (mean $=$ $330 \mathrm{msec}, S D=44.5$ ) than on intentional trials where they freely selected to act (mean $=402 \mathrm{msec}, S D=$ $60.7 ; t(15)=6.04, p<.001)$. This difference may reflect a delay associated with intentional choice, even when the chosen action is motorically identical to the response already prepared. In go/no-go trials of Experiment 1, errors of omission averaged $1.9 \%(S D=2.2)$, and errors of commission averaged 13.9\% (SD $=10.0)$.

\section{Experiment 1: N2 Mean Amplitude}

Because the numbers of trials in each condition were different by design, we avoided bias by analyzing mean rather than peak ERP amplitudes (see Luck, 2005). The N2 ERP component was averaged over a group of frontal-central midline electrodes, Fz, FCz, and Cz, commonly used to measure the N2 component (e.g., Brydges, Anderson, Reid, \& Fox, 2013; Nieuwenhuis et al., 2003;
Bokura et al., 2001; Falkenstein et al., 1999; Pfefferbaum et al., 1985; see Methods for details of this calculation). Figure 2A shows the grand average waveforms for each condition and mean N2 amplitudes.

The ERP showed a negative-going slope during the baseline period, presumably reflecting preparation for the prepotent action. Analysis of N2 mean amplitudes using an ANOVA with Action source (reactive/intentional) and Response (act/inhibit) revealed no significant main effect of Action source $(F(1,15)=2.81, p=.115)$, but a significant main effect of Response $(F(1,15)=17.7, p=$ $.001)$, and a significant interaction $(F(1,15)=27.3$, $p<.001$; Figure 2B). Simple effects $t$ tests showed that reactive no-go signals produced greater N2 amplitudes than reactive go signals $(t(15)=6.12, p<.001$, difference $5.1 \mu \mathrm{V})$, but intentional action and intentional inhibition had similar N2 amplitudes $(t(15)=0.68, p=.683)$. Additional follow-up tests showed that intentional action and inhibition N2 amplitudes were similar to those for reactive no-go signals ( $p=.181, p=.677$ respectively).

\section{Interpreting N2 in Experiment 1: Two Hypotheses}

The key finding from Experiment 1 was a strong N2 component both for intentional choices to act and for intentional choices to inhibit. When choices were instructed, in contrast, the N2 component was found only for reactive inhibition. This pattern of interaction sheds important light on the structure of intentional choice, because the N2 component has been associated with both motor inhibition (e.g., Falkenstein et al., 1999) and with conflict between alternative responses (e.g., Nieuwenhuis et al., 2003). According to an inhibition hypothesis, the $\mathrm{N} 2$ would be present for both intentional go and intentional no-go trials, because the prepotent reactive go response must be either completely or partially suppressed before any intentional selection, whether it
Figure 2. (A) Grand average waveforms for Experiment 1 (averages of $\mathrm{Fz}, \mathrm{FCz}$, and $\mathrm{Cz}$ electrodes), time-locked to onset of imperative target stimulus. Dotted region indicates the N2 component selected for analysis. (B) Mean (standard error) of N2 component in each condition. $\left({ }^{*} p<.05, * * p<.01\right.$, $* * * p<.001)$.

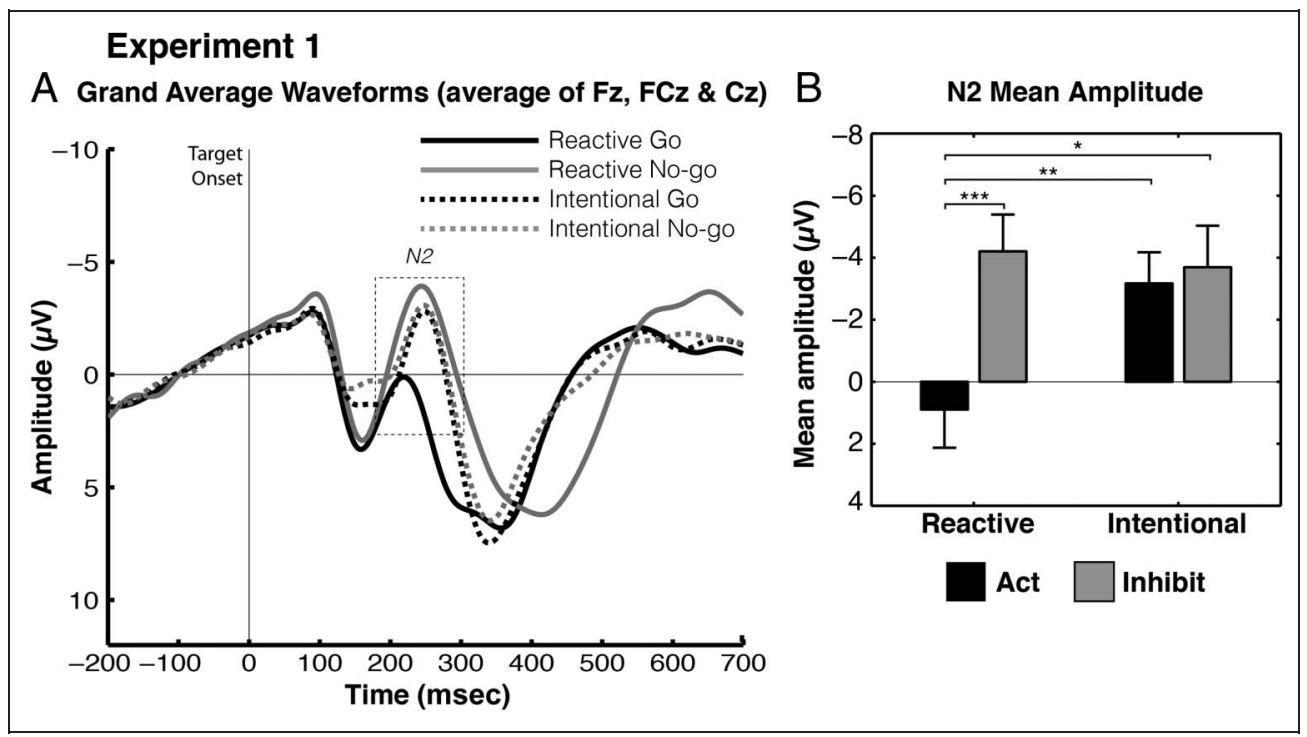


involves the same action or not. That is, the brain terminates the processing of the prepotent action when making a choice, even when one of the choice options involves making the same response voluntarily. This view receives some support from the findings that simple RTs are prolonged, compared to a baseline condition, if participants first prepare to make an action intentionally but then react to the imperative stimulus by making the same response reactively (Obhi \& Haggard, 2004). Furthermore, an irrelevant stop signal that does not actually require inhibition nevertheless produces slowed RTs and inhibitory brain activity (Aron, Behrens, Smith, Frank, \& Poldrack, 2007).

Alternatively, the N2 component may reflect the competition between several alternative responses. Because the reactive go response was three times as frequent (50\%) as the other three conditions, any of those three conditions may trigger a conflict between the prepotent reactive go response and other alternatives. Notice that both the inhibition and the conflict hypothesis imply that intentional go responses are incompatible with reactive go responses, but they differ in the supposed origin of this incompatibility. The inhibition hypothesis treats the incompatibility as a consequence of a fundamental and exclusive competition between intention and reactivity, whereas the conflict hypothesis views it as a by-product of the frequencies of the different stimuli within the experiment.

\section{Experiment 2}

Experiment 2 therefore changed the relative proportions of reactive go and no-go trials to distinguish between the two hypotheses. Now, reactive go trials were $16 \%$, and reactive no-go trials were $50 \%$, reversing the proportions of Experiment 1. Free choice trials remained at 34\%. According to the conflict hypothesis, the rare reactive go trials should now cause the greatest $\mathrm{N} 2$, whereas the most common reactive no-go trials should produce the least. Importantly, response conflict in intentional trials should remain as high as in Experiment 1.

Although it might not be intuitive that a no-go responseessentially a nonaction-can become a prepotent, prepared response option, there is evidence that one can in fact prepare a nonresponse. There is evidence that the distinctive EEG readiness potential, usually observed before a volitional action and associated with response preparation, can also occur before voluntary muscle relaxation, indicating a "nonaction" can be prepared (Terada, Ikeda, Yazawa, Nagamine, \& Shibasaki, 1999; Terada, Ikeda, \& Shibasaki, 1995). Withholding a response involves both pre-SMA and right inferior frontal gyrus involvement (e.g., Aron, 2010). Intriguingly, both fMRI and electrophysiological data have measured pre-SMA and right inferior frontal gyrus activity when participants are asked to prepare response withdrawal (Swann, Cai, Conner, Pieters, \& Michael, 2013). Moreover, there is behavioral evidence that a no-go response can be as nonconsciously primed as the required response, leading to subsequent RT deficits on a go trial (Parkinson \& Haggard, 2014; Hughes, Velmans, \& De Fockert, 2009). Thus, rather than merely being the absence of an action, it seems that a no-go response can be a response in and of itself, which can be internally and externally elicited and prepared. In case of the current Experiment 2, it does seem feasible that an abundant no-go response might become prepotent, and ultimately may evoke response conflict in reactive go and intentional trials, and need to be suppressed to subsequently perform an action.

Conversely, according to the motor inhibition hypothesis, the lower frequency of reactive go trials should make reactive go responses less prepotent and thus greatly reduce the level of motor inhibition required to make any other response. Therefore, the motor inhibition hypothesis predicts that the $\mathrm{N} 2$ component for the reactive no-go, intentional go, and intentional no-go trials should all be greatly reduced.

\section{Results}

Reactive go responses were slower than Experiment 1 (Experiment 2, mean $=399 \mathrm{msec}, S D=44.5$; Experiment 1 , mean $=362.9 \mathrm{msec}, S D=26.2 ; t(1,30)=$ $2.53, p=.017)$, confirming that we successfully manipulated action prepotency. As in Experiment 1, participants in Experiment 2 responded more rapidly on reactive go trials (mean $=399 \mathrm{msec}, S D=44.5$ ) than on intentional go (mean $=409.1 \mathrm{msec}, S D=35.3$; $t(15)=6.05, p<.001)$. In Experiment 2 , the mean rate at which participants freely chose to act in intentional trials was $56.1 \%(S D=6.5)$. Mean miss rate was $3.4 \%$ $(S D=3.4)$, and mean false alarm rate was $1.8 \%$ ( $S D=$ 1.5). An independent samples $t$ test showed that false alarm rates were significantly higher in Experiment 1 than Experiment $2(t(30)=4.66, p<.001)$. This suggests that failures to inhibit in Experiment 2 were fewer than in Experiment 1, which is further evidence that the goal of reducing action prepotency in Experiment 2 was achieved.

\section{Experiment 2: N2 Mean Amplitude}

ERPs from Experiment 2 clearly supported the inhibition hypothesis rather than the conflict hypothesis (Figure 3A and B). ANOVA showed no significant main effects of action source or response and no interaction (all $p s>.1$; see Table 1). To provide further support for null results in Experiment 2, we conducted a Bayes factor analysis on the three pertinent paired-samples comparisons upon which we based our conclusions in Experiment 1 . Bayes factor computations are increasingly used as a statistical method of assessing the reliability of null results (Dienes, 2014). We used an online Bayes 
Figure 3. (A) Grand average waveforms for Experiment 2 (averages of $\mathrm{Fz}, \mathrm{FCz}$, and $\mathrm{Cz}$ electrodes), time-locked to onset of imperative target stimulus. Dotted region indicates the $\mathrm{N} 2$ component selected for analysis. (B) Mean (standard error) of $\mathrm{N} 2$ component in each condition. $(* p<.05, * * p<.01$, $* * * p<.001)$.

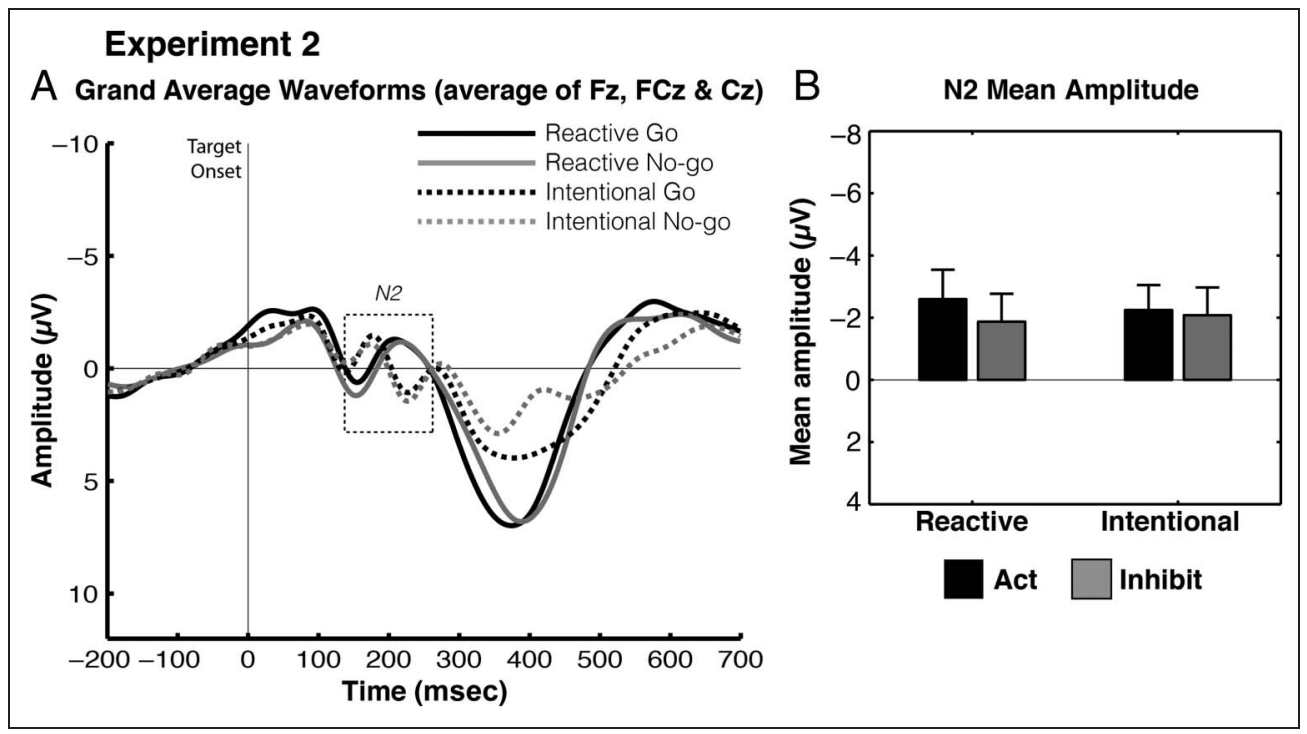

factor calculator (www.lifesci.sussex.ac.uk/home/Zoltan Dienes/inference/Bayes.htm) to calculate Bayes factors for each of the following ERP amplitude comparisons in Experiment 2: reactive go versus reactive no-go; reactive go versus intentional go; reactive go versus intentional no-go. These were the tests that were highly significant in Experiment 1, but nonsignificant in Experiment 2: For each of these tests, we included an a priori expectation of the amplitude difference we might expect to see if the test was significant, which we fixed at a value of $4 \mu \mathrm{V}$, a low rounding of the mean amplitude differences we saw in Experiment 1. All three Bayes factors in Experiment 2 were $\leq 0.15$, well below the sensitivity cutoff value of 0.333 , suggesting that the data support the null (Dienes, 2014).

Thus, in the absence of any strong prepotent drive to act, the need for inhibition in reactive no-go trials was reduced, supporting the inhibition hypothesis. Interestingly, intentional go and intentional no-go trials both showed the same low N2 amplitude as the reactive go and no-go conditions. In contrast, the conflict account predicted a small N2 for the most frequent reactive no-go trials and a large $\mathrm{N} 2$ for reactive go trials and all intentional trials. Our data do not follow this pattern. The fact that there is no difference in $\mathrm{N} 2$ amplitude between reactive and intentional go trials suggests that intentional go responses are only incompatible with reactive go responses when the latter are highly prepotent.

Table 1. Results of ANOVA on N2 Mean Amplitude in Experiment 2

\begin{tabular}{lccc}
\hline Effect & $d f$ & $F$ & $p$ \\
\hline Source (reactive/intentional) & $(1,15)$ & 0.119 & .735 \\
Response (go/no-go) & $(1,15)$ & 0.042 & .840 \\
Source $\times$ Response & $(1,15)$ & 0.113 & .741 \\
\hline
\end{tabular}

\section{DISCUSSION}

To our knowledge, ours is the first electrophysiological study of intentional self-control of action. First, our results suggest that intentional action and intentional inhibition are specific cognitive motor processes that can be distinguished from their reactive counterparts. By integrating free choices to act or inhibit with the conventional go/no-go task, we have been able to directly compare the neural correlates of reactive and intentional inhibition of action.

Our results showed two important differences between intentional action and reactive action, although both classes of action were expressed through identical keypresses. First, RTs were longer following a free choice target stimulus than following a reactive go stimulus. If intentional action merely involved internal triggering of prepared reactive responses, one might expect similar RTs in both situations. The additional delay could partly reflect the time taken to choose between action and inhibition. In addition, the N2 component of the ERP to the target stimulus was much larger for intentional actions than for prepotent reactive actions (in Experiment 1), consistent with a central processing difference. The RT delay for free choices may be associated not only with choosing which intentional response to make but also with inhibiting previously prepared reactions. Importantly, our participants' intentional actions appeared unable to take advantage of the previous preparation to react, although the same motor output was required in both cases. Intentional and reactive systems for action and inhibition thus appeared to use different neural mechanisms, with little sharing of activation between them.

Second, our study sheds light on the neural processes linking instructions to motor responses, in particular, the N2 ERP component. This component was previously suggested to be specific to no-go responses (Bokura et al., 2001; Falkenstein et al., 1999; Eimer, 1993; Pfefferbaum 
et al., 1985) and has been localized variously to ACC and ventral and dorsolateral pFC (Gajewski, Stoerig, \& Falkenstein, 2008; Jonkman, Sniedt, \& Kemner, 2007; Bekker, Kenemans, \& Verbaten, 2004; Lavric, Pizzagalli, \& Forstmeier, 2004; van Veen \& Carter, 2002; Bokura et al., 2001; Kiefer, Marzinzik, Weisbrod, Scherg, \& Spitzer, 1998). However, previous studies could not distinguish whether this component reflected inhibition of the go response or rather a conflict between multiple alternative responses. Our results clearly support the former hypothesis, based on motor inhibition, rather than response conflict. We also show that this component is not specific to no-go responses. The crucial step came from mixing a standard reactive go/no-go paradigm with intentional choices.

The conflict between intentional action and intentional inhibition is identical to the putative conflict between reactive go and reactive no-go. Therefore, conflict accounts of the N2 would suggest no interaction between source of action and go/no-go outcome in Experiment 1, yet a clear interaction was nevertheless found. Furthermore, the high RTs on intentional go trials were consistent with inhibition of the prepotent reactive go response. Thus, N2 was increased in all three conditions where there was behavioral evidence for inhibition of the prepotent response: reactive no-go, intentional go, and intentional no-go. Our results clearly favor an inhibition over a conflict account.

The distinction between a medial system for internally generated action and a lateral system for externally triggered responding is well accepted (Passingham, Bengtsson, \& Lau, 2010). However, few studies have investigated how these two systems may interact. Previous data suggest that the two systems could be mutually inhibitory (Obhi \& Haggard, 2004), except perhaps in the very final period before intentional action (Obhi, Matkovich, \& Chen, 2009). This final period may correspond to activation of the primary motor cortex (Haggard \& Eimer, 1999), on which both internal and external streams for action control must ultimately converge (Sherrington, 1906). Our results are consistent with a fundamental distinction between intentional and reactive action and with an inhibitory link from the intentional to the reactive motor circuit. To act for oneself presupposes inhibition of ongoing stimulus-driven activity. For example, our results indicated that intentional control of action first required intentional inhibition of prepotent stimulus-driven impulse to act. Intentional inhibition could therefore be a fundamental control mechanism for volition in general and not just in inhibitory self-control. Interestingly, early cognitive models of supervisory attention (Norman \& Shallice, 1986) explicitly stated that willed action required suppression of ongoing reactivity but specific evidence of how this might be implement was lacking. Our result provides a long-delayed empirical endorsement of this crucial aspect of willed action.

On the basis of this inhibition model of intentional action decisions, we can make some speculative hypotheses for future research avenues. One main question to ask is, what role, if any, do inhibitory processes have in volitional decisions about action when there is no prepotent motor action that requires suppression? So, for example, what N2 effects would we see if we only presented choice trials? Simply, we might expect replication of the effects seen in Experiment 2, in which N2 amplitudes were reduced overall, with no differences between them, as the prepotency of the go response was greatly reduced. A second question one might ask is, to what extent is the perceptual effort of discriminating the different target stimuli a factor in the RT cost in making an intentional choice and therefore also in the pattern of $\mathrm{N} 2$ results we see? As in many go/no-go paradigms, we use stimuli that are, to some degree, similar to each other, in this case visual arrows that have different directions. Previous research has shown that, in go/no-go paradigms, the amplitude of the $\mathrm{N} 2$ is dependent somewhat on the perceptual overlap between stimuli (Nieuwenhuis, Yeung, \& Cohen, 2004). So, if we used different modalities, visual and auditory, for example, between the reactive go/no-go and intentional targets, would this alter the pattern of N2 effects we see? Such results might also allow us to further disambiguate the roles of motor inhibition per se and those of other perceptual conflict processes in volitional decisions.

One other interesting avenue for further research is to ask what effects occur to inhibitory N2 EEG signals when volitional choices are nonconsciously manipulated. In Parkinson and Haggard (2014), as well as initially developing the go/no-go/choose paradigm, we also demonstrated that RTs and, crucially, the intentional choices themselves can be manipulated by nonconscious subliminal priming: Facilitatory subliminal primes speeded reactive and intentional go responses and also caused participants to make the intentional go action more often, whereas inhibitory primes slowed responses but also made them more likely to choose to withhold the action. Considering the results of the current paper, we can make some predictions regarding an EEG replication of these subliminal priming results: We suggest that the subliminal primes exert nonconscious behavioral effects by modulating the internal inhibitory state of the motor system, which should be indicated by appropriate changes in N2 amplitude. Facilitatory primes should decrease motor inhibition, thus decrease N2 amplitude and speed responses and increase the volitional choice to act, whereas inhibitory primes will produce the opposite effects. This might further elucidate the role of motor inhibition specifically, and motor systems more generally, in the generation of endogenous volitional actions.

In conclusion, we have combined free selection to act or inhibit with reactive go/no-go tasks in an ERP experiment. Our results clearly suggest segregation between the neural systems for internally generated action and for responding to external stimuli, with inhibitory rather than facilitatory interaction between the two systems. Our results also suggest that the intentional choice to act can involve inhibition of a prepared motor response and that this inhibition gives rise to the N2 ERP component. Our 
experiments also clarify the concept of intentional inhibition, as a specific system for intentionally inhibiting a response one is about to make (Filevich et al., 2012), and a key component of volition in general. To this extent, our data are consistent with an endogenous system for braking prepotent motor action, which may be relevant for self-control (Baumeister, Vohs, \& Tice, 2007; Baumeister, Bratslavsky, Muraven, \& Tice, 1998). The human brain has a unique capacity to disengage from stimulus-driven action and to express endogenous action decisions. This could be the neural mechanism underlying philosophical concepts of "free will." Even if an endogenous action decision takes the same motoric form as an externally triggered reaction, volitional inhibition must first stop the tendency to react before any self-generated action can occur. In this sense, society is clearly correct and appropriately aligned with our neurocognitive capacities in allowing an important distinction between deciding for oneself what to do and merely obeying prepotent instructions (Milgram, 1963). Our findings are consistent with these two classes of action being psychologically quite distinct, even when they are behaviorally indistinguishable.

\section{Acknowledgments}

This work was supported by ESRC/ESF ECRP grant to P. H. (Ref: RES-062-23-2183), with additional support from ERC Advanced grant "HUMVOL" and a Templeton Foundation BQFW grant. P. H. was also supported by an ESRC Professorial Fellowship (Ref: ES/J023140/1). The Sackler Centre for Consciousness Science was supported by the Dr. Mortimer and Dame Theresa Sackler Foundation.

Reprint requests should be sent to Jim Parkinson, Sackler Centre for Consciousness Science, University of Sussex, Pevensey 1, Falmer BN1 9QH, United Kingdom, or via e-mail: j.m.parkinson@ sussex.ac.uk.

\section{REFERENCES}

Aron, A. R. (2010). From reactive to proactive and selective control: Developing a richer model for stopping inappropriate responses. Biological Psychiatry, 69, e55-e68.

Aron, A. R., Behrens, T. E., Smith, S., Frank, M. J., \& Poldrack, R. A. (2007). Triangulating a cognitive control network using diffusion-weighted magnetic resonance imaging (MRI) and functional MRI. Journal of Neuroscience, 27, 3743-3752.

Baumeister, R. F., Bratslavsky, E., Muraven, M., \& Tice, D. M. (1998). Ego depletion: Is the active self a limited resource? Journal of Personality and Social Psychology, 74, 1252-1265.

Baumeister, R. F., Vohs, K. D., \& Tice, D. M. (2007). The strength model of self-control. Current Directions in Psychological Science, 16, 351-355.

Bekker, E. M., Kenemans, J. L., \& Verbaten, M. N. (2004). Electrophysiological correlates of attention, inhibition, sensitivity and bias in a continuous performance task. Clinical Neurophysiology, 115, 2001-2013.

Bjork, J. M., \& Gilman, J. M. (2013). The effects of acute alcohol administration on the human brain: Insights from neuroimaging. Neuropharmacology, 84, 101-110.
Boccardi, E., Della Salla, S., Motto, C., \& Spinnler, H. (2002). Utilisation behaviour consequent to bilateral SMA softening. Cortex, 38, 289-308.

Bokura, H., Yamaguchi, S., \& Kobayashi, S. (2001). Electrophysiological correlates for response inhibition in a go/nogo task. Clinical Neurophysiology, 112, 2224-2232.

Brainard, D. H. (1997). The Psychophysics Toolbox. Spatial Vision, 10, 433-436.

Brass, M., \& Haggard, P. (2007). To do or not to do: The neural signature of self-control. Journal of Neuroscience, 27, 9141-9145.

Brydges, C. R., Anderson, M., Reid, C. L., \& Fox, A. M. (2013). Maturation of cognitive control: Delineating response inhibition and interference suppression. PLoS One, 8, e69826.

Della Sala, S., Marchetti, C., \& Spinnler, H. (1991). Right-sided anarchic (alien) hand: A longitudinal study. Neuropsychologia, 29, 1113-1127.

Dienes, Z. (2014). Using Bayes to get the most out of non-significant results. Frontiers in Psycholology, 5, 781.

Donkers, F. C., \& van Boxtel, G. J. (2004). The N2 in go/no-go tasks reflects conflict monitoring not response inhibition. Brain and Cognition, 56, 165-176.

Eimer, M. (1993). Effects of attention and stimulus probability on ERPs in a go/no-go task. Biological Psychology, 35, 123-138.

Enriquez-Geppert, S., Konrad, C., Pantev, C., \& Huster, R. J. (2010). Conflict and inhibition differentially affect the N200/ P300 complex in a combined go/nogo and stop-signal task. Neuroimage, 51, 877-887.

Falkenstein, M., Hoormann, J., \& Hohnsbein, J. (1999). ERP components in go/no-go tasks and their relation to inhibition. Acta Psychologica, 101, 267-291.

Filevich, E., Kühn, S., \& Haggard, P. (2012). Intentional inhibition in human action: The power of 'no'. Neuroscience and Biobehavioral Reviews, 36, 1107-1118.

Gajewski, P. D., Stoerig, P., \& Falkenstein, M. (2008). ERP-Correlates of response selection in a response conflict paradigm. Brain Research, 1189, 127-134.

Haggard, P., \& Eimer, M. (1999). On the relation between brain potentials and the awareness of voluntary movements. Experimental Brain Research, 126, 128-133.

Hughes, G., Velmans, M., \& De Fockert, J. (2009). Unconscious priming of a no-go response. Psychophysiology, 46, 1258-1269.

Jonkman, L. M., Sniedt, F. L. F., \& Kemner, C. (2007). Source localization of the Nogo-N2: A developmental study. Clinical Neurophysiology, 118, 1069-1077.

Kiefer, M., Marzinzik, F., Weisbrod, M., Scherg, M., \& Spitzer, M. (1998). The time course of brain activations during response inhibition: Evidence from event-related potentials in a go/no go task. NeuroReport, 9, 765-770.

Kleiner, M., Brainard, D. H., \& Pelli, D. G. (2007). What's new in Psychtoolbox-3? Perception, 36, ECVP Abstract Supplement.

Kritikos, A., Breen, N., \& Mattingley, J. B. (2005). Anarchic hand syndrome: Bimanual coordination and sensitivity to irrelevant information in unimanual reaches. Brain Research. Cognitive Brain Research, 24, 634-647.

Kühn, S., Haggard, P., \& Brass, M. (2009). Intentional inhibition: How the "veto-area" exerts control. Human Brain Mapping, 30, 2834-2843.

Lavric, A., Pizzagalli, D. A., \& Forstmeier, S. (2004). When 'go' and 'nogo' are equally frequent: ERP components and cortical tomography. European Journal of Neuroscience, 20 , 2483-2488.

Lhermitte, F. (1983). "Utilization behaviour" and its relation to lesions of the frontal lobes. Brain, 106, 237-255.

Libet, B., Gleason, C. A., Wright, E. W., \& Pearl, D. K. (1983). Time of conscious intention to act in relation to onset 
of cerebral activity (readiness-potential). The unconscious initiation of a freely voluntary act. Brain, 106, 623-642.

Luck, S. J. (2005). An introduction to the event-related potential technique. Cambridge, MA: MIT Press.

McBride, J., Sumner, P., Jackson, S. R., Bajaj, N., \& Husain, M. (2013). Exaggerated object affordance and absent automatic inhibition in alien hand syndrome. Cortex, 49, 2040-2054.

Milgram, S. (1963). Behavioral study of obedience. Journal of Abnormal and Social Psychology, 67, 371-378.

Mischel, W., Ebbesen, E. B., \& Zeiss, A. R. (1972). Cognitive and attentional mechanisms in delay of gratification. Journal of Personality and Social Psychology, 21, 204-218.

Nieuwenhuis, S., Yeung, N., \& Cohen, J. D. (2004). Stimulus modality, perceptual overlap, and the go/no-go N2. Psychophysiology, 41, 157-160.

Nieuwenhuis, S., Yeung, N., van den Wildenberg, W., \& Ridderinkhof, K. R. (2003). Electrophysiological correlates of anterior cingulate function in a go/no-go task: Effects of response conflict and trial type frequency. Cognitive, Affective \& Behavioral Neuroscience, 3, 17-26.

Norman, D. A., \& Shallice, T. (1986). Attention to action: Willed and automatic control of behaviour. In R. J. Davidson, G. E. Schwartz, \& D. Shapiro (Eds.), Consciousness and self-regulation advances in research and theory (pp. 1-18). New York: Springer.

Obhi, S. S., \& Haggard, P. (2004). Internally generated and externally triggered actions are physically distinct and independently controlled. Experimental Brain Research, $156,518-523$.

Obhi, S. S., Matkovich, S., \& Chen, R. (2009). Changing the "when" and "what" of intended actions. Journal of Neurophysiology, 102, 2755-2762.

Oostenveld, R., Fries, P., Maris, E., \& Schoffelen, J. M. (2011). FieldTrip: Open source software for advanced analysis of MEG, EEG, and invasive electrophysiological data. Computational Intelligence and Neuroscience, 2011, 156869.

Parkinson, J., \& Haggard, P. (2014). Subliminal priming of intentional inhibition. Cognition, 130, 255-265.
Passingham, R. E., Bengtsson, S. L., \& Lau, H. C. (2010). Medial frontal cortex: From self-generated action to reflection on one's own performance. Trends in Cognitive Sciences, 14, 16-21.

Pelli, D. G. (1997). The VideoToolbox software for visual psychophysics: Transforming numbers into movies. Spatial Vision, 10, 437-442.

Pfefferbaum, A., Ford, J. M., Weller, B. J., \& Kopell, B. S. (1985). ERPs to response production and inhibition. Electroencephalography and Clinical Neurophysiology, 60, 423-434.

Randall, W. M., \& Smith, J. L. (2011). Conflict and inhibition in the cued-go/nogo task. Clinical Neurophysiology, 122, 2400-2407.

Shadlen, M. N., \& Gold, J. I. (2004). The neurophysiology of decision-making as a window on cognition. In M. S. Gazzaniga (Ed.), The cognitive neurosciences (3rd ed., pp. 1229-1241). Cambridge, MA: Elsevier.

Sherrington, C. S. (1906). The integrative action of the nervous system. New Haven: Yale University Press, 1947 edition.

Smith, J. L., Smith, E. A., Provost, A. L., \& Heathcote, A. (2010). Sequence effects support the conflict theory of N2 and P3 in the go/nogo task. International Journal of Psychophysiology, 75, 217-226.

Swann, N. C., Cai, W., Conner, C. R., Pieters, T. A., \& Michael, P. (2013). Roles for the pre-supplementary motor area and the right inferior frontal gyrus in stopping action: Electrophysiological responses and functional and structural connectivity. Neuroimage, 59, 2860-2870.

Terada, K., Ikeda, A., \& Shibasaki, H. (1995). Movement-related cortical potentials associated with voluntary muscle relaxation. Electroencephalography and Clinical Neurophysiology, 95, 335-345.

Terada, K., Ikeda, A., Yazawa, S., Nagamine, T., \& Shibasaki, H. (1999). Movement-related cortical potentials associated with voluntary relaxation of foot muscles. Clinical Neurophysiology, 110, 397-403.

van Veen, V., \& Carter, C. S. (2002). The timing of actionmonitoring processes in the anterior cingulate cortex. Journal of Cognitive Neuroscience, 14, 593-602. 